\title{
microRNA-15 Activates NF-kB Pathway via Down Regulating Expression of Adenosine A2 Receptor in Ulcerative Colitis
}

\author{
Huixia Zhang ${ }^{a}$ Wei Li ${ }^{b}$ \\ aDepartment of Gastroenterology, The First Affiliated Hospital of Zhengzhou University, Zhengzhou, \\ bDepartment of Physiology and neurobiology, School of Basic Medicine Sciences, Zhengzhou \\ University, Zhengzhou, China
}

\section{Key Words}

Ulcerative colitis $\cdot$ miRNA-15 $•$ A2aAR $・ N F-k B$

\begin{abstract}
Background/Aims: The role of miR-15 in ulcerative colitis (UC) is unclear. In this study, we found that miR-15 downregulated the expression of adenosine A2a receptor (A2aAR) in the colonic tissues of patients with UC and in HT-29 human colonic epithelial cells. Methods: The study population comprised patients with UC $(n=23)$, irritable bowel syndrome (IBS, $n=22)$, and healthy subjects $(n=20)$. The levels of miR-15, A2aAR, and protein in colon tissue biopsies were determined by real-time quantitative reverse transcription polymerase chain reaction, immunofluorescence staining, and Western blotting. The TargetScan prediction algorithm was used to identify the miR-15 binding site in the 3'-UTR of A2aAR. To assess the effects of miR-15 on A2aAR levels, HT-29 cells were transfected with miR-15 mimics. Results: Relative to expression in healthy subjects, A2aAR expression was decreased in UC patients and was similar in IBS patients. MiR-15 levels were higher in UC patients than in IBS patients or healthy subjects. A2aAR was the target of miR-15 in HT-29 cells, which downregulated A2aAR mRNA levels. MiR-15 mimics induced nuclear translocation of NF-KB p65 and upregulated the expression of IL-8 and IFN- $\gamma$ in colonic epithelial cells; these effects were reversed by an miR-15 inhibitor. A2aAR knockdown confirmed that miR-15 activated the NF-KB cascade. Conclusion: Our data suggest that miR-15 modulates inflammatory and immune responses by suppressing the expression of $A 2 a A R$ and regulating the NF- $\mathrm{kB}$ cascade.
\end{abstract}

\section{Introduction}

(C) 2018 The Author(s)

Published by S. Karger AG, Basel

Irritable bowel syndrome (IBS) is a relapsing inflammatory disorder of the gastrointestinal tract that can lead to the development of Crohn's disease or ulcerative colitis (UC). An abnormal immune response and aberrant expression of various genes are involved in the pathogenesis of UC $[1,2]$. 


\section{Cellular Physiology Cell Physiol Biochem 2018;51:1932-1944

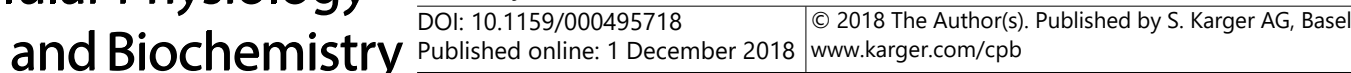 \\ Zhang et al.: microRNA-15 Regulates Expression of A2aAR in Ulcerative Colitis}

MicroRNAs (miRNAs) are single-stranded non-coding RNA molecules of about 23-24 nucleotides that regulate target gene expression by binding to their 3 '-untranslated regions (UTRs) $[3,4]$. Thousands of miRNAs have been identified that regulate the expression of approximately $30 \%$ of human genes [5]. MiR-126, miR-155, and miR-150 are upregulated in UC and induce inflammation via the NF- $\mathrm{KB}$ signalling cascade [6-8]. Upregulation of the NF- $\kappa B$ pathway in UC-affected tissues induces the transcription of interleukin-8 (IL-8), interferon- $\gamma(\mathrm{IFN}-\gamma)$, and interleukin-1 $\beta$ (IL-1 $\beta$ ) [9-11]. The expression of other miRNAs is downregulated in UC tissues, and miR-19a and miR-192 modulate the expression of TNF- $\alpha$ and MIP- $2 \alpha$, respectively $[12,13]$. In chronic lymphocytic leukaemia, miR-15 and miR-16 induce apoptosis via the Bcl-2 pathway [14]. Ageilan et al. [15] reported that miR15a and miR16 act as tumour suppressors by targeting WNT3A, BCL2, CCND1, and MCL1, and that the expression of both miRNAs is downregulated in prostate cancer and pituitary adenoma. However, the role of miR15 in UC is unclear.

Adenosine is released by metabolically active cells and is important in various cellular processes. Adenosine modulates inflammation by binding to the A1, A2a, A2b, and A3 receptors $[16,17]$. Stimulation of the A2a adenosine receptor (A2aAR) activates adenylate cyclase,[16] which is expressed in human intestinal cells [18, 19]. Elevated levels of extracellular adenosine leads to activation of A2aAR, which suppresses inflammation in rats with UC [16]. Selective A2aAR agonists reportedly suppress inflammation via the NF$\kappa B$ pathway, $[18,19]$ and $A 2 b A R$ expression is downregulated by miR-128a25 and miR-27b [20]. The expression of miR-15 and miR-214 is inversely correlated with A2aAR levels in polymorphonuclear leukocytes [21]. Therefore, the effects of miR-15 on A2aAR expression via the NF- $\kappa B$ cascade in UC-affected colon tissue warrants further investigation.

In this study, we evaluated the levels of miR-15 and A2aAR in UC-affected colon tissue and analysed the regulation of A2aAR expression by miR-15 in human colonic epithelial cells. The results demonstrated that miR-15 regulates the NF- $\kappa B$ pathway by modulating A2aAR expression in UC-affected colon tissue.

\section{Materials and Methods}

\section{Subjects and specimens}

Sixty-five subjects who underwent colonoscopic screening in 2016 at the School of Basic Medicine Sciences, Zhengzhou University (Zhengzhou, China) were enrolled. The study population comprised patients with active UC $(n=23)$, IBS $(n=22)$, and healthy subjects $(n=20)$. IBS was diagnosed based on Rome III criteria, [22] namely, recurring abdominal pain for at least 3 consecutive days/month for 3 months accompanied by at least two of the following: improvement in the elimination of stools, change in stool frequency, and change in stool form. To distinguish IBS from simple changes in bowel habits, the gut symptoms had to be of at least 3 months' duration with an onset of 6 months before diagnosis and with no signs of metabolic, inflammatory, or cancerous processes that could explain the symptoms.

Colon tissue samples were collected from subjects who had not undergone a therapeutic procedure for at least 3 months. The tissue samples were taken from the sites of inflammation in the colon, and all subjects provided written informed consent for use of their samples. UC activity was graded using the UC disease activity index (UCDAI),[23] which includes stool frequency, associated bleeding, and the presence of colon lining in stools. Symptoms were graded on a scale of 0 to 3, with 3 being the highest. The total UCDAI score was $0-10$ and scores were categorised as follows: $0-2$, remission; $3-6$, mild UC; 7-10, moderate UC; and $>10$, severe UC. Only patients with a UCDAI score $>7$ were enrolled in this study. The tissue samples were dissected to remove any connective tissue, stored in liquid nitrogen at the tissue bank of The First Affiliated Hospital of Zhengzhou University, and coded according to the guidelines of the Ethics Committee. The study was approved by the Ethics Committee (Reference No. 878004ZU/HEC). 


\section{Cellular Physiology Cell Physiol Biochem 2018;51:1932-1944

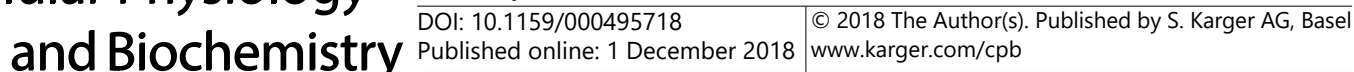

\section{Cell lines, culture conditions, and reagents}

HT-29 cells were used because they enable recapitulation of the in vivo environment, and they have a similar proteome as cells in human intestinal scrapings. HT-29 cells are also more stable during culture than other UC cell lines [24, 25]. The cell lines were obtained from the Microbiology Department of our hospital, and their use was approved by the Ethics Committee. The cells were cultured in Roswell Park Memorial Institute medium (Sigma-Aldrich, St. Louis, MO, USA) supplemented with foetal bovine serum (Sigma), streptomycin $(100 \mu \mathrm{g} / \mathrm{mL})$, and penicillin $(100 \mathrm{IU} / \mathrm{mL})$ at $37^{\circ} \mathrm{C}$ under humidified conditions in a $5 \% \mathrm{CO}_{2}$ atmosphere. The universal miRNA inhibitor (inhibitor negative control), miR-15 mimics, miR-15 inhibitor, universal miRNA mimics (mimic negative controls), universal siRNA negative control, and A2aAR siRNA were purchased from Sigma. Phosphate-buffered saline (PBS) was also obtained from Sigma.

\section{Immunofluorescence staining of tissue sections}

Colon tissue sections ( $5 \mu \mathrm{m}$ thickness) were subjected to immunofluorescence staining (IFS). Briefly, the sections were treated with Triton X-100 $(0.1 \%)$ for 20 min to increase their permeability, blocked in donkey serum albumin (10\%) for 20 min, and incubated with rabbit anti-human A2aAR Iry antibody for $12 \mathrm{~h}$ at $4^{\circ} \mathrm{C}$. Then the tissue sections were washed three times in PBS and incubated for $60 \mathrm{~min}$ with a fluorescein isothiocyanate-conjugated donkey anti-rabbit IIry antibody in the dark at room temperature. The sections were subsequently washed three times in PBS. The negative control (lacking the anti- $\mathrm{I}^{\mathrm{ry}}$ antibody) was prepared using an identical procedure. Nuclei were counterstained with 4',6-diamidino-2-phenylindole, and the sections were coverslipped using fluorescent mounting medium and observed under an inverted fluorescence microscope (Olympus, Tokyo, Japan).

\section{RNA Extraction and real-time quantitative reverse transcriptase polymerase chain reaction}

Total miRNA was extracted using an RNA isolation kit (Thermo Fisher Scientific, Waltham, MA, USA) following the manufacturer's protocol and converted into cDNA using a miScript RT Kit. Real-time quantitative reverse transcriptase polymerase chain reaction (qRT-PCR) was performed using the Cells Direct $^{\text {TM }}$ One-Step qRT-PCR Kit (Thermo Fisher Scientific). Gene expression was normalised to that of endogenous U6, and A2aAR, IL-8, and IFN- $\gamma$ mRNA levels were normalised to those of GAPDH as a loading control. The sequences of the miRNA primers (Applied Biosystems ${ }^{\mathrm{TM}}$, Foster City, CA, USA) are listed in Table 1.

\section{Transfection of HT-29 cells with miRNA inhibitors and mimics}

HT-29 cells were cultured for $12 \mathrm{~h}$ at room temperature $(\sim 90 \%$ confluence $)$ and were transfected $\left(5 \times 10^{5}\right)$ with an miR-15 inhibitor, miR-15 mimics, and negative controls $(50,100$, and $150 \mathrm{nM})$ using Lipofectamine reagent (Thermo Fisher Scientific) per the manufacturer's instructions.

\section{Transfection and Renilla and Firefly} luciferase assays

After PCR amplification, A2aAR 3 '-UTR sequences were cloned into the pRL Renilla Luciferase Control Reporter Vector (Fitchburg, WI, Promega). The PCR primer (Table 2) used to construct wild-type A2aAR 3'-UTR was named pmiR-A2aAR-wt. Its mutant, pmiR-

Table 1. The sequences of primers used in the study

\begin{tabular}{lcc}
\hline For mRNA qPCR & Direction & Name of Primer (5'-3') \\
\hline A2aAR & Forward & AAGGAGGGCAAGAACCACTC \\
& Reverse & AGCACACAGGCAAAGAAGTTG \\
IFN- $\gamma$ & Forward & AATGTCCAACGCAAAGCAAT \\
& Reverse & AGCATCTGACTCCTTTTCGC \\
IL-8 & Forward & GCAGAGGGTTGTGGAGAAGT \\
& Reverse & AACCCTACAACAGACCCACA \\
GAPDH & Forward & GGGTGTGAACCATGAGAAGT \\
& Reverse & CAGTGATGGCATGGACTGTG \\
qPCR primer & Reverse & miScript RT kit \\
miR-15 & Forward & UAGCAGCACAUAAUGGUUUGUG \\
U6 SnRNA & Forward & CTCGCTTCGGCAGCACA \\
\hline
\end{tabular}

A2aAR-mut, was generated by replacing the miR-15-binding site in A2aAR-3'UTR-mut-R by site-directed mutagenesis. HT-29 cells were transfected using Lipofectamine reagent with miR15 mimics or inhibitor (150 nM),
Table 2. The primers for PCR selected to construct the A2aAR $3^{\prime}$-UTR

\begin{tabular}{lc}
\hline Name & PCR primers \\
\hline A2aAR-3' UTR-wt-F & $5^{\prime}$-GCGGCCTCGAGTCCTGATGATTCATGGAGTTT-3' \\
A2aAR-3' UTR-wt-R, & $5^{\prime}$-AATGCGGCCGCTCAGACGCCATTCTCATTT-3' \\
A2aAR-3'UTR-mut-F & $5^{\prime}$-GTCGGTCCACGACGAT ACCTGGCACCAGAGCCTC-3' \\
A2aAR-3' UTR-mut-R & $5^{\prime}$-TGCCAGGTATCGTCGTGGACCGACGGC AGACCCA-3' \\
\hline
\end{tabular}




\section{Cellular Physiology Cell Physiol Biochem 2018;51:1932-1944 \begin{tabular}{l|l|l} 
and Biochemistry Published online: 1 December 2018 & $\begin{array}{l}\text { (c) } 2018 \text { The Author(s). Published by S. Karger AG, Basel } \\
\text { www.karger.com/cpb }\end{array}$ \\
\hline
\end{tabular} \\ Zhang et al.: microRNA-15 Regulates Expression of A2aAR in Ulcerative Colitis}

pmiR-A2aAR-mutant (500 ng), pmiR-A2aAR-wt (500 ng), or the negative control. Renilla and Firefly luciferase assays were conducted $48 \mathrm{~h}$ after transfection using a Dual Luciferase ${ }^{\circledR}$ System (Promega corp. USA) according to the manufacturer's instructions. The experiment was performed in triplicate.

\section{TNF- $\alpha$ stimulation}

HT-29 cells were cultured in 6-well plates and incubated at room temperature in 5\% $\mathrm{CO}_{2}$ for $12 \mathrm{~h}$ ( $\sim 90 \%$ confluence). Then the cells were transfected with the negative control $(150 \mathrm{nM})$, miR-15 mimics, or miR-15 inhibitor (150 nM). Subsequently, the cells were transfected with the negative control siRNA (150 $\mathrm{nM})$, A2aAR-siRNA (150 nM), or the miR-15 inhibitor (150 nM). After incubation for $48 \mathrm{~h}$, the cells were treated with TNF- $\alpha$ for 12 or $24 \mathrm{~h}$.

\section{Western blotting}

To extract total protein, HT-29 cells and colonic tissues were treated with cell lysis buffer (Cell Signaling Technology, Danvers, MA USA) followed by NE-PER ${ }^{\mathrm{TM}}$ Nuclear and Cytoplasmic Extraction Reagents (Thermo Fisher Scientific) to extract nuclear and cytoplasmic proteins. The total protein content was estimated using a protein assay kit (bicinchoninic acid method; Elabscience®, Bethesda, MD, USA). The proteins were separated by sodium dodecyl sulphate-polyacrylamide gel electrophoresis (SDS-PAGE, 12\%) and equal quantities were transferred to Durapore ${ }^{\circledR}$ polyvinylidene fluoride (PVDF) membranes (Sigma). The PVDF membranes were blocked in fat-free milk (5\%) and incubated at $4^{\circ} \mathrm{C}$ for $12 \mathrm{~h}$ with rabbit anti-human A2aAR $\mathrm{I}^{\mathrm{ry}}$ antibody (diluted 1:600), anti-human mouse NF- $\kappa \mathrm{B}$ p65 antibody (diluted 1:500), and rabbit anti-human H3 antibody (diluted 1:1000) (all from Abcam, Cambridge, MA, USA). The membranes were rinsed with Tris buffer containing Tween-20 and incubated for 60 min with an antiII $^{\text {ry }}$ antibody. Signals were detected using an electrochemiluminescence reagent, recorded on films (Kodak, Rochester, NY, USA), and evaluated using Quantity One software.

\section{Enzyme-linked immunosorbent assay \\ After centrifugation to} remove cell debris, IL-8 and IFN- $\gamma$ protein levels in HT-29 cell culture supernatants were determined in triplicate via the enzyme-linked immunosorbent assay (ELISA) using an ELISA kit (Abcam) and ELISA reader.

\section{Results}

A2aAR expression in colonic epithelial cells

IFS for A2aAR in colon tissue from all of the subjects showed the presence of A2aAR in colonic epithelial cells (Fig. 1). A2aAR expression in colonic epithelial cells was similar in healthy subjects and IBS patients, but was lower in UC patients.

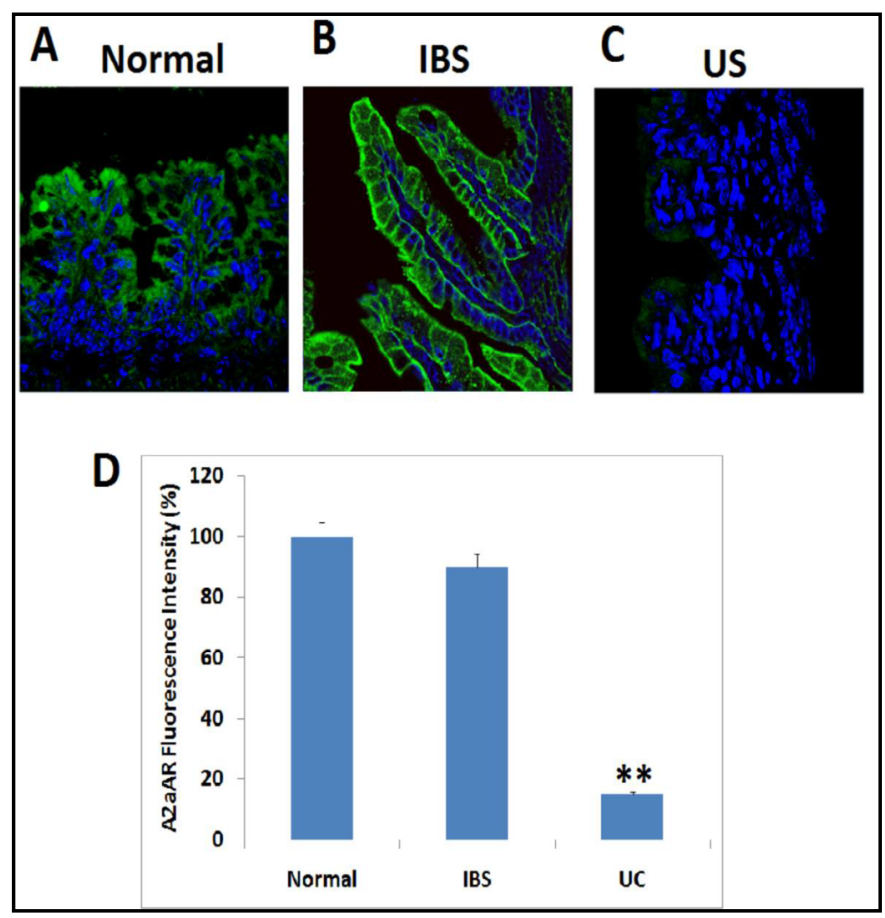

Fig. 1. Immunofluorescence staining studies of tissues obtained from colons of patients, the labeling of A2aAR and DAPI was done using specific antibodies. The nuclei's were counter stained blue whereas green fluorescence signal indicated A2aAR (A-C). Staining of A2aAR was mainly present in epithelial cells and was present majorly in biopsies of normal (A) and IBS (B) subjects, whereas the expression decreased in tissue biopsies of UC subjects (C). Quantification of A2aAR immunofluorescence. All the data presented are mean \pm SEM $(n=23$ for active UC; $n=22$ and $n=20$ for Normal subjects). 


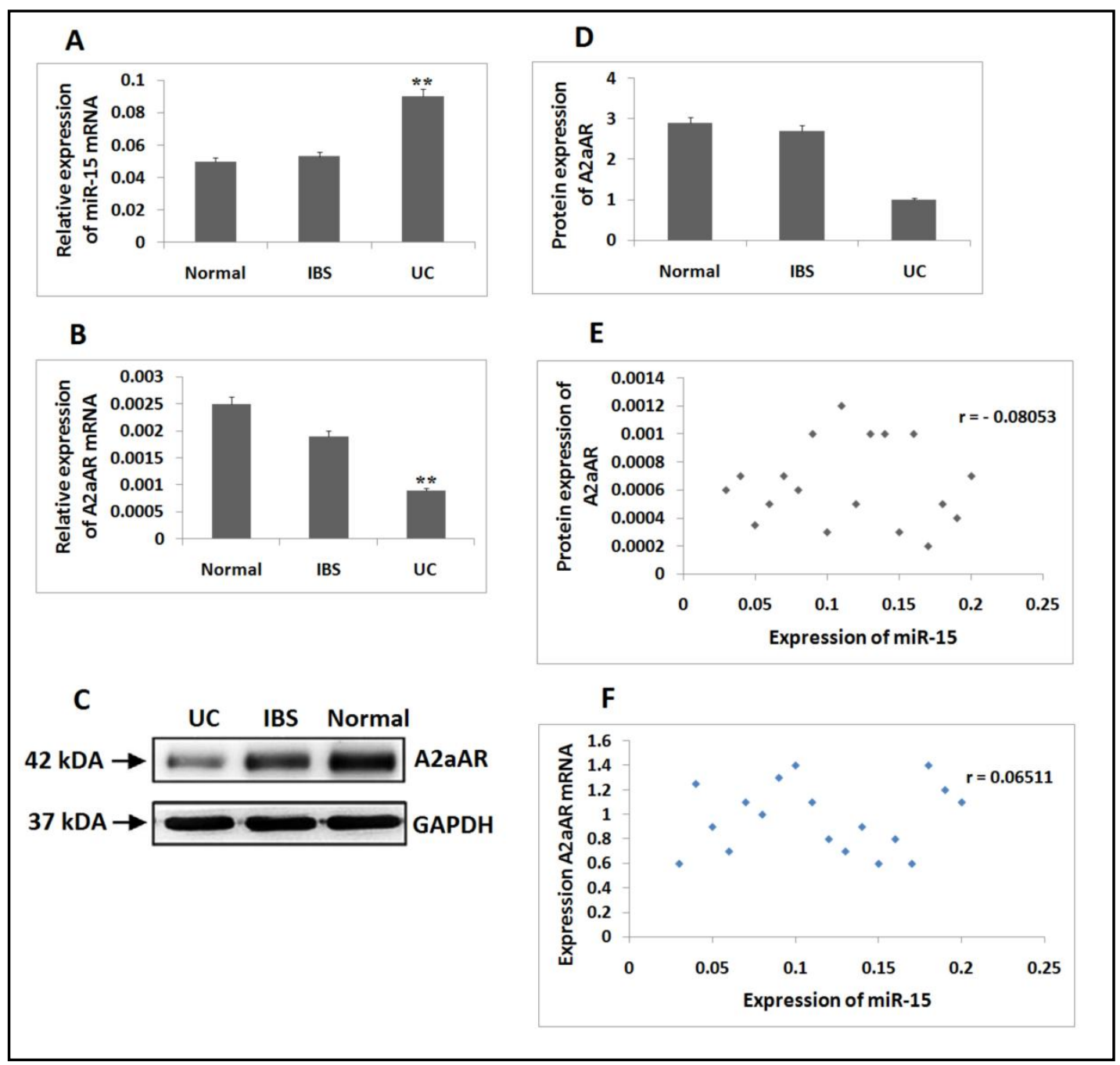

Fig. 2. (A) qRT-PCR analysis for Expression of miR-15 in tissues, (B) expression of A2aAR mRNA in sigmoid colon tissues UC $(n=23)$, IBS $(n=22)$ and healthy subjects $(n=20)$ (Data is mean $+S D, * * P<0.01)$. (C) Expression of proteins by western blot for expression of A2aAR in colon tissues using GAPDH is loading control (Data is mean $+\mathrm{SD}$, ${ }^{* *} \mathrm{P}<0.01$ ). (D) Correlation was established between miR-15 and expression of A2aAR protein. (E) We found that expression of miR-15 depicted a strongly negative correlation with levels of A2aAR protein in tissues of UC subjects $(n=23$, Pearson correlation $r=-0.08053, P<0.05)$. (F) Expression of miR-15 and expression of A2aAR mRNA in tissues biopsies of patients with ulcerative colitis (Pearson correlation $r=0.06511, \mathrm{P}>0.05$ ).

\section{mRNA levels of miR-15 and A2aAR in colon tissue}

The protein and mRNA levels of A2aAR and miR-15 in colon tissue were determined by Western blotting and qRT-PCR, respectively. The level of miR-15 was higher in colon tissue from UC patients (Fig. 2A) than from IBS patients and healthy subjects $(\mathrm{P}<0.01)$. However, A2aAR mRNA levels were significantly lower in colon tissue from UC patients (Fig. 2B-D) than from IBS patients and healthy subjects $(P<0.01)$. The levels of A2aAR and miR-15 in colon tissue did not markedly differ between IBS patients and healthy subjects $(P>0.05)$. IFS yielded comparable results, with the exception of a significant inverse correlation between the levels of miR-15 and A2aAR (Fig. 2E) ( $\mathrm{r}=-0.08053$, $\mathrm{P}<0.05)$. There were no significant associations between A2aAR and miR-15 mRNA levels in the colon tissue of UC patients (Fig. 2F; $r=0.06511, \mathrm{P}>0.05$ ). 


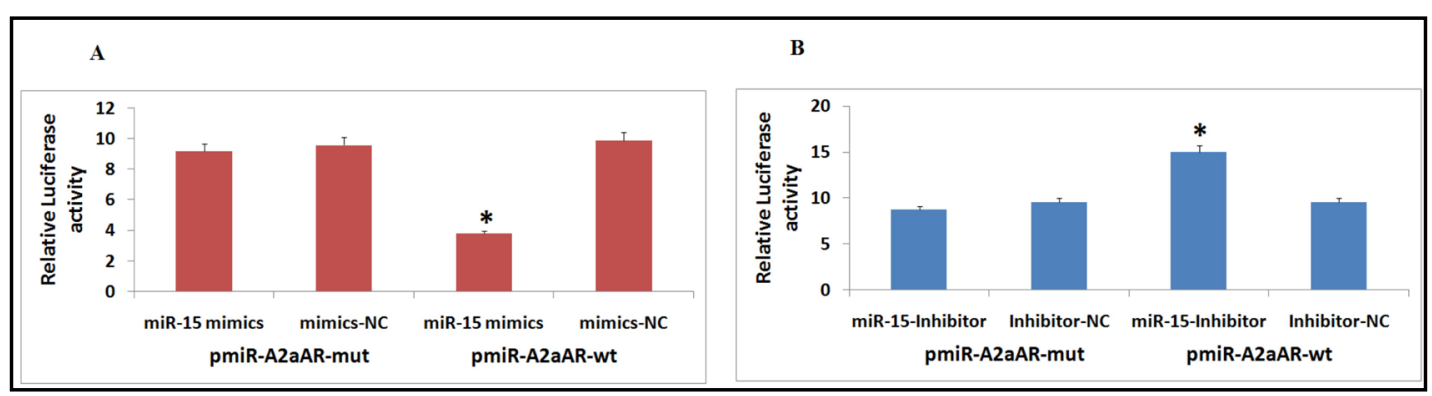

Fig. 3. (A) The Renilla luciferase activity was measured after HT-29 cells were co-transfected with miR15 mimics, miR-15-mimics-neagtive control, pmiR-A2aAR-wild type and pmiR-A2aAR-mut vector. (B) The Renilla luciferase activity was measured after HT-29 cells were co-transfected with miR-15 inhibitor, miR15-inhibitor-neagtive control, pmiR-A2aAR-wild type and pmiR-A2aAR-mut vector. All the results are mean, standard deviation, ${ }^{*} \mathrm{P}<0.05$ versus all the other groups.

\section{Identification of the $3^{\prime}-U T R$ of A2aAR as the miR-15-binding site}

Next, we identified the miR-15-binding site in the 3'-UTR of A2aAR using the TargetScan prediction algorithm. To determine whether miR-15 targets A2aAR mRNA, a luciferase reporter vector harbouring the wild-type A2aAR 3'-UTR sequence downstream of pmiR-A2aAR-wt and pmiR-A2aAR-mut was constructed. Luciferase assays of HT-29 cells transfected with pmiR-A2aAR-mut or pmiR-A2aAR-wt and miR-15 mimics showed that transfection of miR-15 mimics significantly decreased the luciferase activity of pmiR-A2aARwt compared to transfection of the mimic negative controls $(\mathrm{P}<0.05$, Fig. $3 \mathrm{~A})$. Moreover, the miR-15 inhibitor resulted in a significant increase in the luciferase activity of cells transfected with pmiR-A2aAR-wt compared to those transfected with the inhibitor negative control (Fig. 3B, $\mathrm{P}<0.05$ ). The luciferase activity of pmiR-A2aAR-mut was not affected by transfection with miR-15 mimics or the miR-15 inhibitor $(P>0.05)$. These data show that A2aAR is a target of miR-15 in HT-29 cells.

\section{Effects of miR-15 on endogenous A2aAR levels}

The mRNA level of miR-15 was significantly increased (Fig. 4A; $\mathrm{P}<0.01$ ), and that of A2aAR was decreased in a dose-dependent manner by transfection of HT-29 cells with miR-15 mimics compared to miR-15 mimic negative controls (Fig. 4B; $\mathrm{P}<0.05$ ). A2aAR protein levels in transfected cells mirrored those of mRNA (Fig. 4B-D; P < 0.05). By contrast, transfection of HT-29 cells with the miR-15 inhibitor significantly reduced the levels of miR-15 (Fig. 4E) $(\mathrm{P}<0.01)$, whereas A2aAR protein and mRNA levels were upregulated by $50 \%$ compared to cells transfected with the inhibitor negative control (Fig. $4 \mathrm{~F}-\mathrm{H} ; \mathrm{P}<0.05$ ). Therefore, miR-15 downregulates A2aAR expression in HT-29 cells.

\section{Effects of miR-15 on pro-inflammatory cytokines and NF- $\kappa B$ signalling}

To evaluate the relationship between miR-15 and the NF- $\kappa B$ cascade, we assessed NF- $\kappa B$ activation in TNF- $\alpha$-treated HT-29 cells. TNF- $\alpha$ treatment resulted in increased cytoplasmic and nuclear NF- $\kappa \mathrm{B}$ p65 protein levels (Fig. $5 \mathrm{~A}, \mathrm{~B} ; \mathrm{P}<0.05$ ), showing that TNF- $\alpha$ induced the nuclear translocation of NF- $\kappa \mathrm{B}$ p65. Moreover, TNF- $\alpha$ treatment resulted in increased levels of the pro-inflammatory cytokines IL-8 and IFN- $\gamma$ (Fig. 5C-F; P < 0.05). To investigate the role of miR-15 in the NF- $\kappa B$ cascade, HT- 29 cells were transfected with miR-15 mimics or mimic negative controls $(150 \mathrm{nM})$. Transfection of miR-15 mimics resulted in significant increases and decreases in nuclear and cytoplasmic NF- $\kappa \mathrm{B}$ p65 levels, respectively, compared to transfection of mimic negative controls (Fig. 6A, B; P $<0.05$ ). Similarly, the levels of IL-8 and IFN- $\gamma$ were upregulated in cells transfected with miR-15 mimics compared to those transfected with mimic negative controls (Fig. 6C-F; P < 0.05). Moreover, the miR15 inhibitor increased and decreased the cytoplasmic and nuclear levels of NF- $\kappa B$ p65, respectively, compared to the inhibitor negative control (Fig. 6A, B; P < 0.05). Similarly, 

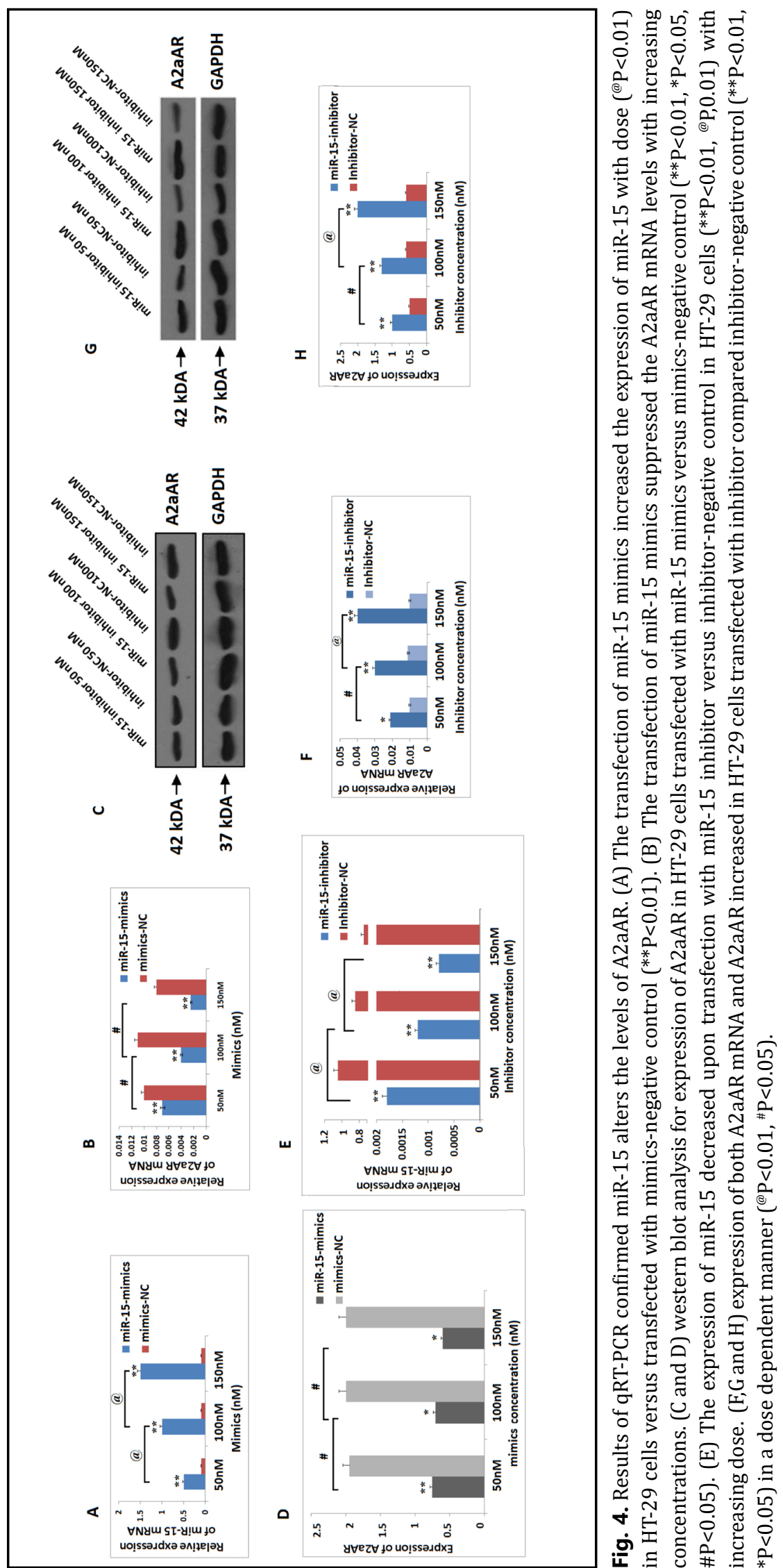
the levels of IL-8 and IFN- $\gamma$ were markedly lower in HT-29 cells transfected with the miR15 inhibitor compared to those transfected with the inhibitor negative control (Fig. 6C-F; $\mathrm{P}<0.05$ ). Therefore, miR-15 induced IL-8 and IFN- $\gamma$ expression and activated the NF- $\mathrm{KB}$ pathway in HT-29 cells.

\section{miR-15 activates the $N F-\kappa B$ pathway by regulating A2aAR expression}

Our findings suggested that miR-15 decreased the expression of A2aAR and activated the NF- $\mathrm{BB}$ pathway. Similarly inhibition of miR-15 enhanced the expression of A2aAR and suppressed the activation of NF- $\kappa B$. Next, we evaluated the effects of a miR-15 inhibitor and A2aAR siRNA on the expression of A2aAR. A2aAR mRNA levels were lower in HT-29 cells transfected with A2aAR siRNA than in those transfected with siRNA negative control. Co-transfection of TNF- $\alpha$-treated cells with a miR-15 inhibitor and A2aAR siRNA resulted in the increased nuclear translocation of NF- $\mathrm{BB}$ p65 and upregulation of IL-8 and IFN- $\gamma$

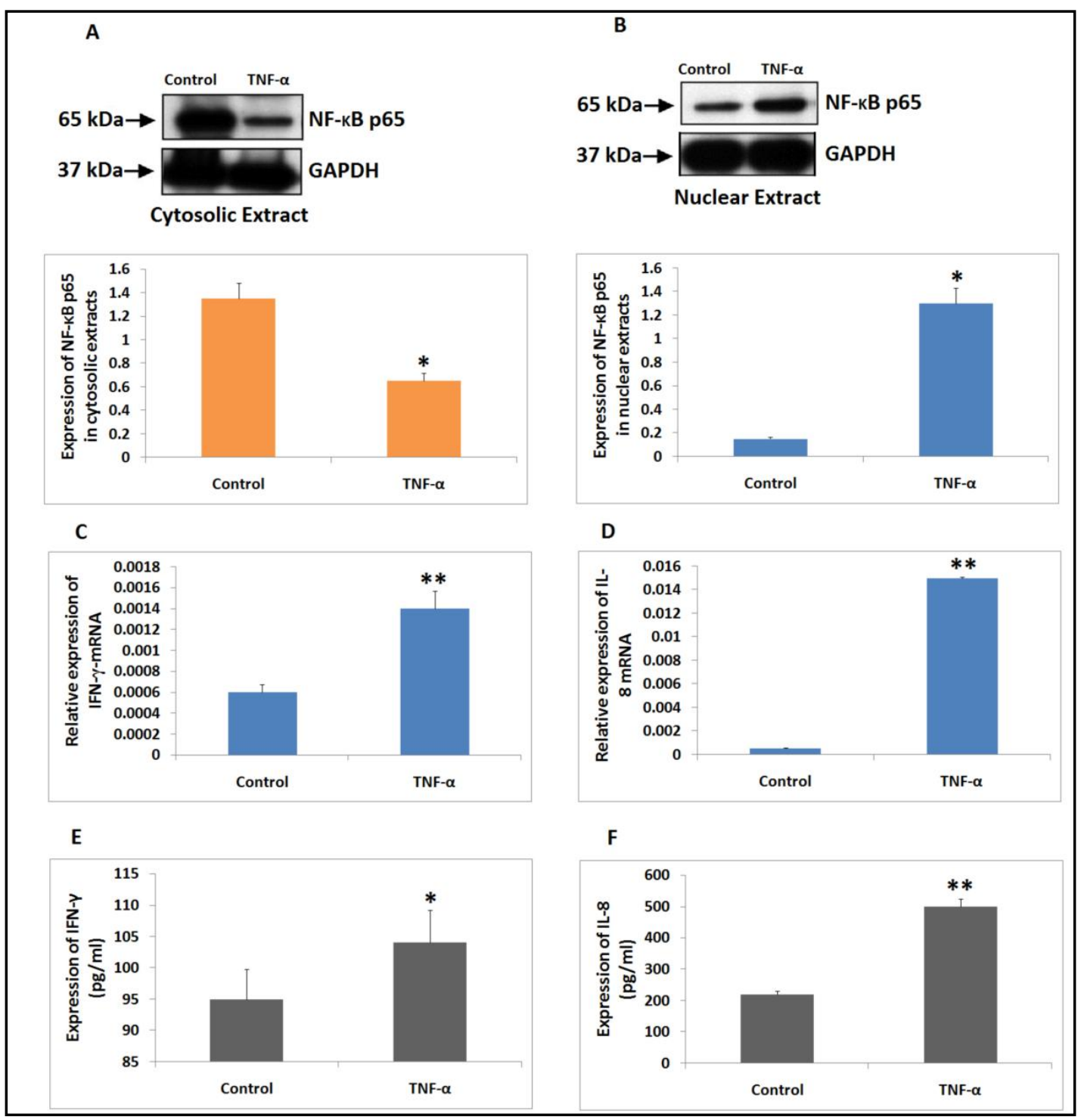

Fig. 5. Western blot analysis for expression of NF- $\kappa B$ p65 from cytosolic and nuclear extracts in control and TNF- $\alpha$ treated HT-29 cells for 12 and 24 h (A-B).qRT-PCR analysis for (C and D) IL-8mRNA and IFN- $\gamma$ levels in HT-29 cells. ELISA analysis for (E and F) IL-8 and IFN- $\gamma$ from supernatants of HT-29 cells. Data presented is mean, $\mathrm{SD}\left({ }^{*} \mathrm{P}<0.05,{ }^{* *} \mathrm{P}<0.01\right.$ compared to controls). 


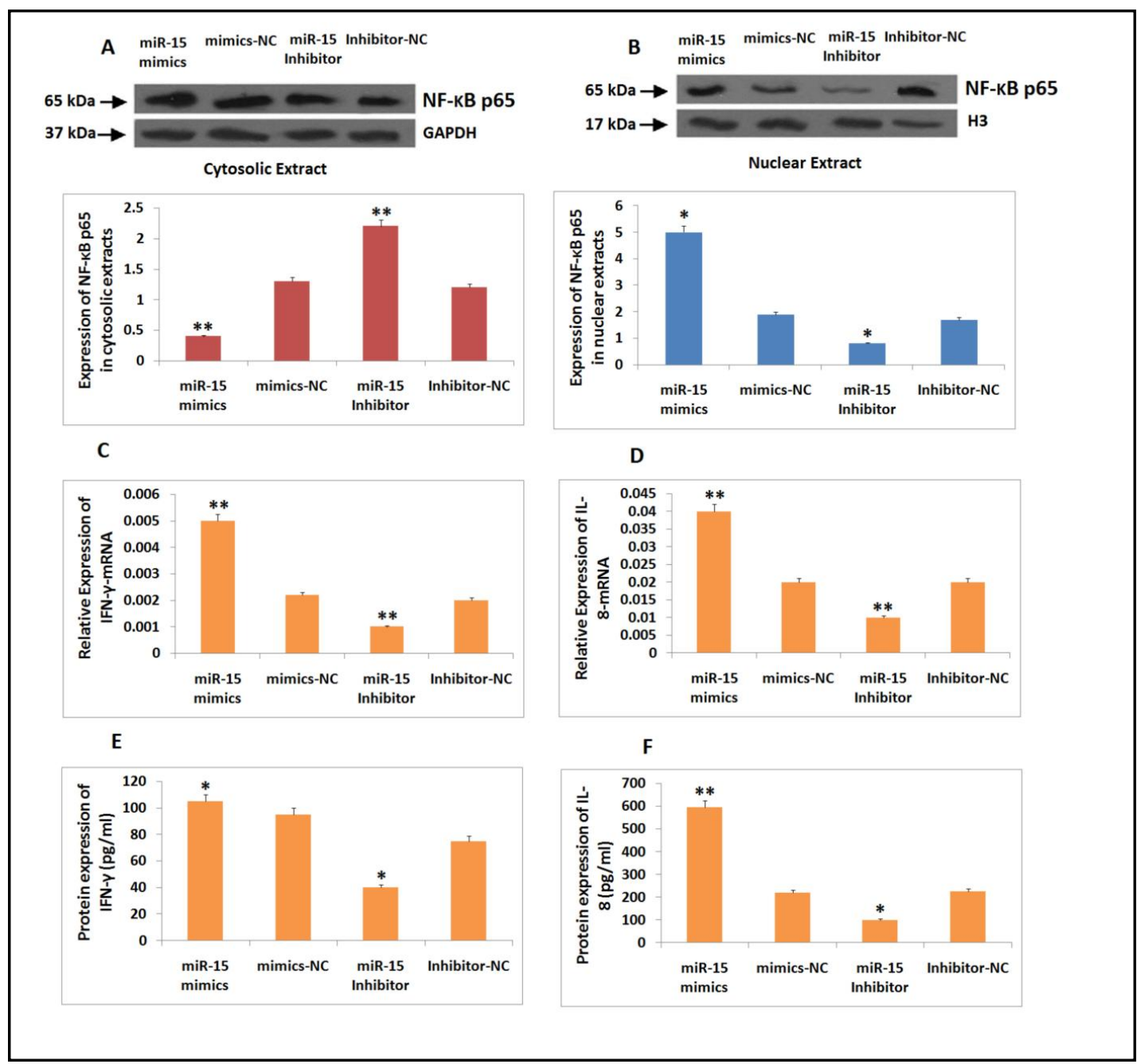

Fig. 6. miR-15 increased the translocation of NF- $\kappa B$ p 65 protein in nucleus whereas decreased in cells transfected with miR-15 inhibitor (A-B). miR-15 mimics increased whereas miR-15 inhibitor decreased levels of IFN- $\gamma$,IL-8 and IFN- $\gamma$-mRNA, IL-8-mRNA significantly compared to mimics-negative control and inhibitor-negative control respectively (C-F). Data are presented as mean, $\mathrm{SD}\left({ }^{*} \mathrm{P}<0.05,{ }^{* *} \mathrm{P}<0.01\right.$ versus corresponding negative control.

compared to cells transfected with the siRNA negative control and an miR-15 inhibitor (Fig. $7 \mathrm{~A}-\mathrm{F} ; \mathrm{P}<0.05)$. Therefore, siRNA-mediated downregulation of A2aAR reversed the effects of the miR-15 inhibitor via TNF- $\alpha$-mediated activation of the NF- $\kappa B$ pathway. Thus, miR-15 activates the NF- $\kappa B$ pathway by regulating the expression of $A 2 \mathrm{aAR}$. 


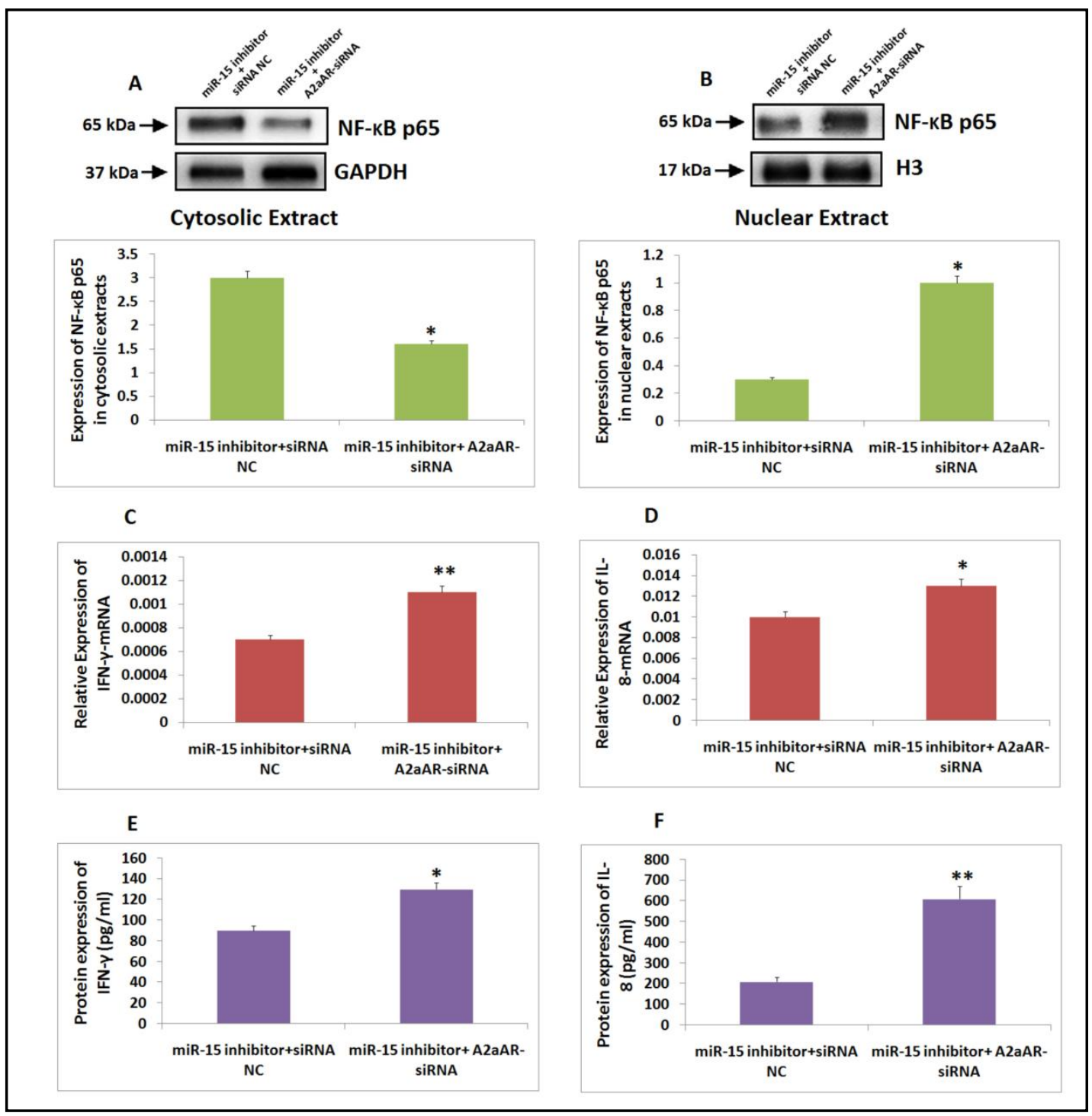

Fig. 7. Expression of NF- $\kappa \mathrm{B}$ p65 decreased in cytosolic extract were as increased in nuclear extracts after treating cells with miR-15 inhibitor and A2aAR-siRNA (A,B). (C-F) The levels of IFN- $\gamma$,IL-8, IFN- $\gamma-$ mRNA and IL-8-mRNA increased significantly in cells treated with miR-15 inhibitor and A2aAR-siRNA compared to miR-15 inhibitor and siRNA-negative control. $\left({ }^{*} \mathrm{P}<0.05,{ }^{* *} \mathrm{P}<0.01\right.$ compared siRNA-negative control).

\section{Discussion}

MiRNAs have been implicated in the physiopathology of abdominal disorders such as IBS and UC. MiR-15 reportedly induces apoptosis [14] and acts as a tumour suppressor by modulating the expression of WNT3A, BCL2, CCND1, and MCL1 [15]. However, its role in UC is unclear. In this study, we evaluated miR-15 levels in colonic tissue from IBS patients, UC patients, and healthy subjects. The levels of miR-15 in colon tissues from UC patients were higher than those in colon tissues from IBS patients and healthy subjects, suggesting that altered levels of miR-15 are linked to IBS. MiR-15 regulates TNF- $\alpha$-mediated apoptosis in

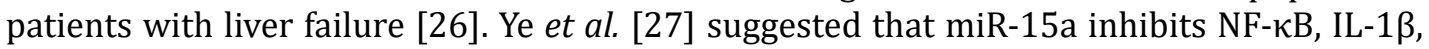
and TNF $\alpha$ signalling in retinal endothelial cells. A recent report suggested that the $3^{\prime}$-UTR of A2aAR harbours binding sites for miR-15 and miR-16 [21]. Thus, miR-15 may interfere with pro-inflammatory signalling by modulating gene expression. 


\section{Cellular Physiology Cell Physiol Biochem 2018;51:1932-1944

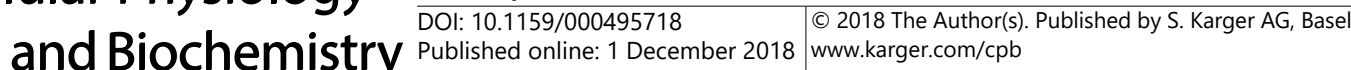

A2aAR regulates several biological processes including inflammation. Upregulation of A2aAR reportedly suppressed inflammation in rats with colitis [28]. Therefore, we evaluated the role of A2aAR in UC. In this study, A2aAR expression in colon tissue was lower in UC patients than in healthy subjects. A2aAR levels were inversely related to those of miR-15 in UC patients (Fig. 2A, D, F). In addition, luciferase assays suggested that the 3 '-UTR of A2aAR is a target of miR-15. Analyses using miR-15 mimics and a miR-15 inhibitor showed that miR-15 downregulated the expression of A2aAR in HT-29 cells.

$\mathrm{NF}-\kappa \mathrm{B}$ is a protein complex responsible for controlling gene transcription, and its p65 subunit plays important roles in inflammation and the immune response. In response to stimuli, NF- $\mathrm{BB}$ p65 translocates from the cytoplasm to the nucleus where it modulates the expression of numerous genes including those encoding cytokines, mediators of inflammation, and chemokines [29]. It also modulates the molecular networks responsible for cellular processes associated with IBS [30]. Yang et al. [31] suggested that activation of A2aAR suppresses the NF- $\kappa \mathrm{B}$ cascade in smooth muscle cells. To investigate the correlation between miR-15 and the NF- $\kappa B$ pathway, an miR-15 inhibitor and miR-15 mimics were transfected into TNF- $\alpha$-treated HT- 29 cells. The miR-15 mimics increased the expression of miR-15, which suggested enhanced nuclear translocation of NF- $\mathrm{B}$ p65 and upregulation of IL-8 and IFN- $\gamma$ expression. By contrast, the miR-15 inhibitor upregulated A2aAR expression and inhibited NF- $\kappa B$ activity (Fig. 6). These findings are in agreement with prior reports $[27,32]$. Moreover, knockdown of A2aAR by transfection with miR-15 and A2aAR-siRNA induced the nuclear translocation of NF- $\kappa$ B p65, resulting in upregulation of IL-8 and IFN- $\gamma$ expression.

\section{Conclusion}

Taken together, our results suggest that miR-15 downregulates A2aAR and activates the $\mathrm{NF}-\kappa \mathrm{B}$ cascade. Therefore, miR-15 is involved in the pathogenesis of UC by downregulating the expression of $\mathrm{A} 2 \mathrm{aAR}$ via the NF- $\mathrm{BB}$ pathway. In conclusion, miR-15 and A2aAR are candidate therapeutic targets in UC patients.

\section{Disclosure Statement}

The authors report no conflicts of interest in this work.

\section{References}

1 Podolsky DK: Inflammatory bowel disease. N Engl J Med 2002;347:417-429.

- Farrell RJ, Peppercorn MA: Ulcerative colitis: Lancet 2002;359:331-340.

-3 Bartel DP: MicroRNAs: Target recognition and regulatory functions. Cell 2009;136:215-233.

4 He L, Hannon GJ: MicroRNAs: small RNAs with a big role in gene regulation. Nat Rev Genet 2004;5:522531.

5 Nana-Sinkam SP, Croce CM: Clinical applications for microRNAs in cancer. Clin Pharmacol Ther 2013;93:98104.

6 Feng X, Wang H, Ye S, Guan J, Tan W, Cheng S, Wei G, Wu W, Wu F, Zhou Y: Up-Regulation of microRNA-126 May Contribute to Pathogenesis of Ulcerative Colitis via Regulating NF-kappaB Inhibitor Iк B $\alpha$. Plos One 2012;7:e52782.doi:10.1371/journal.pone.0052782.

7 Bian Z, Li L, Cui J, Zhang H, Liu Y, Zhang CY, Zen K: Role of miR-150-targeting c-Myb in colonic epithelial disruption during dextran sulphate sodium-induced murine experimental colitis and human ulcerative colitis. J Pathol 2011;225:544-553.

-8 Singh UP, Murphy AE, Enos RT, Shamran HA, Singh NP, Guan H: miR-155 deficiency protects mice from experimental colitis by reducing T helper type 1/type 17 responses. Immunology 2014;143:478-489. 


\section{Cellular Physiology Cell Physiol Biochem 2018;51:1932-1944 \begin{tabular}{l|l|l|l|l}
\hline DOI: 10.1159/000495718 & C 2018 The Author(s). Published by S. Karger AG, Basel \\
and Biochemistry
\end{tabular}

-9 Wei J, Feng J: Signaling pathways associated with inflammatory bowel disease. Recent Pat Inflamm Allergy Drug Discov 2010;4:105-117.

10 Wullaert A, Bonnet MC, Pasparakis M: NF-kappaB in the regulation of epithelial homeostasis and inflammation. Cell Res 2011;21:146-158.

-11 Spehlmann ME, Eckmann L: Nuclear factor-kappa B in intestinal protection and destruction. Curr Opin Gastroenterol 2009;25:92-99.

12 Wu F, Zhang S, Dassopoulos T, Harris ML, Bayless TM, Meltzer SJ, Brant SR, Kwon JH: Identification of microRNAs associated with ileal and colonic Crohn's disease. Inflamm Bowel Dis 2010;16:1729-1738.

13 Wu F, Zikusoka M, Trindade A, Dassopoulos T, Harris ML, Bayless TM, Brant SR, Chakravarti S, Kwon JH: MicroRNAs are differentially expressed in ulcerative colitis and alter expression of macrophage inflammatory peptide-2 alpha. Gastroenterology 2008;135:1624-1635.

14 Cimmino A, Calin GA, Fabbri M, Iorio MV, Ferracin M, Shimizu M, Wojcik SE, Aqeilan RI, Zupo S, Dono M, Rassenti L, Alder H, Volinia S, Liu CG, Kipps TJ, Negrini M, Croce CM: miR-15 and miR-16 induce apoptosis by targeting BCL2. Proc Natl Acad Sci U S A 2005;102:13944-13949.

15 Aqeilan RI, Calin GA, Croce CM: miR-15a and miR-16-1 in cancer: discovery, function and future perspectives. Cell Death Differ 2010;17:215-220.

16 Ye JH, Rajendran VM: Adenosine: An immune modulator of inflammatory bowel diseases. World J Gastroenterol 2009;15:4491-4498.

17 Hasko G, Linden J, Cronstein B, Pacher P: Adenosine receptors: therapeutic aspects for inflammatory and immune diseases. Nat Rev Drug Discov 2008;7:759-770.

18 Antonioli L, Fornai M, Colucci R, Awwad O, Ghisu N, Tuccori M, Del Tacca M, Blandizzi C: Differential recruitment of high affinity $\mathrm{A} 1$ and $\mathrm{A} 2 \mathrm{~A}$ adenosine receptors in the control of colonic neuromuscular function in experimental colitis. Eur J Pharmacol 2011;650:639-649.

-19 Michael S, Warstat C, Michel F, Yan L, Müller CE, Nieber K: Adenosine A(2A) agonist and A(2B) antagonist mediate an inhibition of inflammation-induced contractile disturbance of a rat gastrointestinal preparation. Purinergic Signal 2010;6:117-124.

20 Kolachala VL, Wang L, Obertone TS, Prasad M, Yan Y, Dalmasso G, Gewirtz AT, Merlin D, Sitaraman SV: Adenosine 2B receptor expression is post-transcriptionally regulated by microRNA. J Biol Chem 2010;285:18184-18190.

-21 Heyn J, Ledderose C, Hinske LC, Limbeck E, Möhnle P, Lindner HA, Kreth S: Adenosine A2A receptor upregulation in human PMNs is controlled by miRNA-214, miRNA-15 and miRNA-16. Shock 2012;37:156163.

22 Longstreth GF, Thompson WG, Chey WD, Houghton LA, Mearin F, Spiller RC: Functional bowel disorders. Gastroenterology 2006;130:1480-1491.

-23 Sutherland LR, Martin F, Greer S, Robinson M, Greenberger N, Saibil F, Martin T, Sparr J, Prokipchuk E, Borgen L: 5-Aminosalicylic acid enema in the treatment of distal ulcerative colitis, proctosigmoiditis, and proctitis. Gastroenterology 1987;92:1894-1898.

24 Lipps C, May T, Hauser H, Wirth D: Eternity and functionality-rational access to physiologically relevant cell lines. Biol Chem 2013;394:1637-1648.

-25 Zweibaum A, Laburthe M, Grasset E, Louvard D: Use of cultured cell lines in studies of intestinal cell differentiation and function. Compr Physiol (Suppl 19) 2011;223-255.

-26 An F, Gong B, Wang H, Yu D, Zhao G, Lin L, Tang W, Yu H, Bao S, Xie Q: miR-15b and miR-16 regulate TNF mediated hepatocyte apoptosis via BCL2 in acute liver failure. Apoptosis 2012;17:702-716.

-27 Eun-Ah Ye, Li Liu, Jiang Y, Jan J, Gaddipati S, Suvas S, Steinle JJ: miR-15a/16 reduces retinal leukostasis through decreased pro-inflammatory signaling. J Neuroinflammation. 2016;13:305.

28 Ren T, Tian T, Feng X, Ye S, Wang H, Wu W, Qiu Y, Yu C, He Y, Zeng J, Cen J, Zhou Y: An adenosine A3 receptor agonist inhibits DSS-induced colitis in mice through modulation of the NF-kappaB signaling pathway. Sci Rep. 2015;5:9047-9055.

29 Tang Y, Clayburgh DR, Mittal N, Goretsky T, Dirisina R, Zhang Z, Kron M, Ivancic D, Katzman RB, Grimm G, Lee G, Fryer J, Nusrat A, Turner JR, Barrett TA: Epithelial NF-kappaB enhances transmucosal fluid movement by altering tight junction protein composition after T cell activation. Am J Pathol.2010;176:158-167. 


\section{Cellular Physiology Cell Physiol Biochem 2018:51:1932-1944

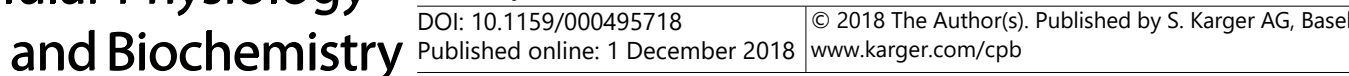 \\ Zhang et al.: microRNA-15 Regulates Expression of A2aAR in Ulcerative Colitis}

30 Singh K, Chaturvedi R, Barry DP, Coburn LA, Asim M, Lewis ND, Piazuelo MB, Washington MK, Vitek MP, Wilson KT: The apolipoprotein E-mimetic peptide COG112 inhibits NF-kappaB signaling, proinflammatory cytokine expression, and disease activity in murine models of colitis. J Biol Chem 2011;286:3839-3850.

31 Yang J, Zheng X, Haugen F, Darè E, Lövdahl C, Schulte G, Fredholm BB, Valen G: Adenosine increases LPSinduced nuclear factor kappa B activation in smooth muscle cells via an intracellular mechanism and modulates it via actions on adenosine receptors. Acta physiol(Oxf) 2014;210:590-599.

-32 Liang X, Xu Z, Yuan M, Zhang Y, Zhao B, Wang J, Zhang A, Li G: MicroRNA-16 suppresses the activation of inflammatory macrophages in atherosclerosis by targeting PDCD4. Int J Mol Med 2016;37:967-975. 